\title{
Evaluation of MicroScan MIC Panels for Detection of Oxacillin-Resistant Staphylococci
}

\author{
GAIL L. WOODS* AND PAUL YAM \\ Department of Pathology and Microbiology, University of Nebraska Medical Center, 42nd and Dewey Avenue, \\ Omaha, Nebraska 68105
}

Received 14 October 1987/Accepted 19 January 1988

\begin{abstract}
Clinical isolates of staphylococci (420 Staphylococcus aureus isolates and 248 coagulase-negative staphylococci) were tested by both MicroScan MIC panels (MicroScan, West Sacramento, Calif.) and an oxacillin agar screen (Mueller-Hinton agar [Difco Laboratories, Detroit, Mich.] containing $6 \mu \mathrm{g}$ of oxacillin per ml and 4\% $\mathrm{NaCl})$ to evaluate the ability of MicroScan to detect oxacillin-resistant strains. MicroScan panels and oxacillin agar screen plates were incubated at $35^{\circ} \mathrm{C}$ for $24 \mathrm{~h}$ and at $30^{\circ} \mathrm{C}$ for an additional $24 \mathrm{~h}$. Endpoints were recorded at 24 and $48 \mathrm{~h}$. By MicroScan, $23(5.5 \%)$ and $30(7 \%) S$. aureus isolates and $161(65 \%)$ and $162(65 \%)$ coagulase-negative staphylococci were oxacillin resistant at 24 and 48 h, respectively. At both 24 and 48 h, 23 $(5.5 \%) S$. aureus isolates and $162(65 \%)$ coagulase-negative staphylococci were resistant by the oxacillin agar screen. Five strains for which the oxacillin MIC was 2 or $4 \mu \mathrm{g} / \mathrm{ml}$ and eight strains resistant to oxacillin only at $48 \mathrm{~h}$ were further evaluated by broth macrodilution testing for oxacillin with and without clavulanic acid, by oxacillin and amoxicillin-clavulanic acid disk diffusion, and by oxacillin agar screen comparing MuellerHinton agars purchased from Difco and BBL Microbiology Systems, Cockeysville, Md. By this additional testing, all $10 \mathrm{~S}$. aureus isolates and 1 of 3 coagulase-negative staphylococci examined produced increased amounts of $\beta$-lactamase. One coagulase-negative staphylococcus appeared to be truly intermediately oxacillin susceptible. There was no significant difference in the rate of detection of oxacillin resistance between MicroScan and the agar screen. MicroScan panels should be incubated for $24 \mathrm{~h}$ only, because prolonged incubation caused strains producing excessive amounts of $\beta$-lactamase to appear to be falsely oxacillin resistant.
\end{abstract}

Methicillin-resistant Staphylococcus aureus has been a significant problem in the United States since the mid-1970s $(8,13)$. In recent years, coagulase-negative staphylococci have been recognized as important nosocomial pathogens $(2$, $7,12,20,21)$; therefore, the detection of methicillin resistance in these organisms is also a concern. Because most methicillin-resistant strains are heterogeneous and are recognized only after specific manipulations of culture conditions, various recommendations have been made to enhance their in vitro detection $(18,19,22)$. One such method is the oxacillin agar screen (Mueller-Hinton agar [MHA] containing $4 \% \mathrm{NaCl}$ and $6 \mu \mathrm{g}$ of oxacillin per $\mathrm{ml}, 10 \mu \mathrm{g}$ of methicillin per $\mathrm{ml}$, or $6 \mu \mathrm{g}$ of nafcillin per $\mathrm{ml}$ and incubated at $35^{\circ} \mathrm{C}$ for $24 \mathrm{~h}$ [19]). For MIC testing, the National Committee for Clinical Laboratory Standards standard M7-A (16) recommends testing staphylococci with the following specific modifications: (i) when oxacillin, methicillin, and nafcillin are tested, the broth should be supplemented with $2 \% \mathrm{NaCl}$; (ii) the inoculum should be prepared directly from overnight growth on an agar plate; and (iii) incubation should be for a full $24 \mathrm{~h}$ at $35^{\circ} \mathrm{C}$. Disk diffusion testing should be performed according to National Committee for Clinical Laboratory Standards guidelines (15). Other recommendations have included incubation at $30^{\circ} \mathrm{C}(10)$ and incubation for $48 \mathrm{~h}(4$, 6).

Many hospital microbiology laboratories, however, use automated rather than the above-described conventional methods of susceptibility testing. It is important, therefore, that these automated systems be able to reliably detect methicillin-resistant staphylococci. MicroScan has changed

* Corresponding author. its MIC panels over the past few years. The earlier panels contained methicillin, which was later replaced with nafcillin. In studies comparing detection of methicillin-resistant staphylococci by these early MicroScan panels and by conventional reference methods, the sensitivity of MicroScan ranged from $80 \%$ with methicillin to about $90 \%$ with nafcillin (a MIC of $>1 \mu \mathrm{g} / \mathrm{ml}$ was considered to indicate resistance) at $24 \mathrm{~h}(1,3,9,17)$. There have been few studies examining the present MicroScan panels, which contain oxacillin in broth supplemented with $2 \% \mathrm{NaCl}(5 ; \mathrm{A}$. $\mathrm{H}$. Brenner, J. H. Picklo, and S. M. Joern, Abstr. Annu. Meet. Am. Soc. Microbiol. 1987, C109, p. 341). We therefore prospectively tested 668 clinical isolates of staphylococci by MicroScan MIC panels and the oxacillin agar screen to determine the ability of the MicroScan MIC panels to detect oxacillin (methicillin)-resistant $S$. aureus and coagulasenegative staphylococci.

\section{MATERIALS AND METHODS}

Organisms. Clinical isolates of staphylococci from all body sites were tested. Of 668 strains, 420 were identified as $S$. aureus by the tube coagulase test (23). The 248 coagulasenegative staphylococci were not further identified. After initial testing, all isolates were stored in sterile defibrinated sheep blood at $-70^{\circ} \mathrm{C}$. If further testing was required, organisms were subcultured twice on 5\% sheep blood agar. The second transfer was used for all testing.

Antimicrobial susceptibility testing. (i) MicroScan MIC panels. Microdilution susceptibility testing was performed according to manufacturer instructions. Briefly, MicroScan MIC panels (MicroScan, West Sacramento, Calif.) were stored at $-70^{\circ} \mathrm{C}$ and allowed to thaw at room temperature 
just before use. From an 18- to 24 -h blood agar plate, isolated colonies were emulsified in $0.85 \%$ sterile saline to yield a turbidity equal to the density of a $0.5 \mathrm{McF}$ arland standard. The suspension was vortexed, and $0.5 \mathrm{ml}$ was added to $25 \mathrm{ml}$ of sterile distilled water containing $0.02 \%$ Tween 80 . Panels were inoculated by using the MicroScan inoculator set. The final inoculum ranged from $1 \times 10^{5}$ to $5 \times 10^{5} \mathrm{CFU}$ per well, which was confirmed by colony counts periodically throughout the study. Panels were incubated for $24 \mathrm{~h}$ at $35^{\circ} \mathrm{C}$, and if after incubation for $24 \mathrm{~h}$ the oxacillin MIC was $\leq 4 \mu \mathrm{g} / \mathrm{ml}$, panels were reincubated at $30^{\circ} \mathrm{C}$ for an additional $24 \mathrm{~h}$. Endpoints were recorded at 24 and $48 \mathrm{~h}$. Appropriate control organisms were also tested.

(ii) Oxacillin agar screen. MHA (Difco Laboratories, Detroit, Mich.) containing $6 \mu \mathrm{g}$ of oxacillin (Beecham Laboratories, Bristol, Tenn.) per $\mathrm{ml}$ and $4 \% \mathrm{NaCl}$ was inoculated with approximately $10^{4} \mathrm{CFU}$. The inoculum was prepared by suspending staphylococci from an 18- to 24-h blood agar plate in $0.85 \%$ sterile saline to yield a density equal to that of a $0.5 \mathrm{McF}$ arland standard. The agar plates were then spot inoculated with a cotton swab that had been dipped in the suspension (C. Thornsberry, Letter, Antimicrob. Newsl. $1: 43-50,1984)$. Plates were incubated at $35^{\circ} \mathrm{C}$ for $24 \mathrm{~h}$, and if the strain was susceptible to oxacillin after incubation for 24 $\mathrm{h}$, it was incubated for an additional $24 \mathrm{~h}$ at $30^{\circ} \mathrm{C}$. Control strains of oxacillin-resistant and -susceptible $S$. aureus were tested on each plate.

(iii) Broth macrodilution. Susceptibility of the following strains to oxacillin with and without $4 \mu \mathrm{g}$ of clavulanic acid (Beecham) per ml was tested by broth macrodilution: (i) isolates that were resistant to oxacillin by MicroScan only after incubation for $48 \mathrm{~h}$ (seven $S$. aureus isolates and one coagulase-negative staphylococcus) and (ii) other isolates for which the oxacillin MIC was 2 or $4 \mu \mathrm{g} / \mathrm{ml}$ by MicroScan at 24 h (three $S$. aureus isolates and two coagulase-negative staphylococci). One isolate of $S$. aureus for which the oxacillin MIC was $4 \mu \mathrm{g} / \mathrm{ml}$ could not be recovered after storage at $-70^{\circ} \mathrm{C}$. Broth macrodilution was performed according to National Committee for Clinical Laboratory Standards guidelines (16) with cation-supplemented MuellerHinton broth (Remel, Lenexa, Kans.) containing $2 \% \mathrm{NaCl}$ and incubation at $35^{\circ} \mathrm{C}$ for $24 \mathrm{~h}$. Serial twofold dilutions of oxacillin ranging from 0.006 to $100 \mu \mathrm{g} / \mathrm{ml}$ were tested. Methicillin-resistant (ATCC 43300) and methicillin-susceptible (oxacillin MIC, 2 to $4 \mu \mathrm{g} / \mathrm{ml}$ ) (ATCC 43387) strains of $S$. aureus served as controls.

(iv) Disk diffusion. Disk diffusion testing of the susceptibility of the above-described 13 strains to oxacillin and amoxicillin-clavulanic acid was performed according to $\mathrm{Na}$ tional Committee for Clinical Laboratory Standards guidelines (15). The inoculum was prepared directly from 18-h agar plates, and MHA plates (Remel) were incubated at $35^{\circ} \mathrm{C}$ for $24 \mathrm{~h}$. Controls included S. aureus ATCC 43387 and $S$. aureus ATCC 43300.

(v) Oxacillin agar screen MHA comparison. By. using the previously described method, the above-indicated 13 staphylococcal strains were retested by oxacillin agar screen to compare MHAs obtained from Difco and BBL Microbiology Systems, Cockeysville, Md. Control strains tested were $S$. aureus ATCC 43300 and S. aureus ATCC 43387.

\section{RESULTS}

The activity of oxacillin against staphylococci as determined by MicroScan MIC panels and the results of the oxacillin agar screen after incubation for 24 and $48 \mathrm{~h}$ are shown in Table 1 . With an oxacillin MIC of $\geq 4 \mu \mathrm{g} / \mathrm{ml}$ representing resistance, $23(5.5 \%)$ strains of $S$. aureus were resistant by MicroScan after incubation for $24 \mathrm{~h}$, and $30(7 \%)$ strains were resistant at $48 \mathrm{~h}$. Of the coagulase-negative staphylococci, $161(65 \%)$ and $162(65 \%)$ strains were oxacillin resistant by MicroScan after incubation for 24 and $48 \mathrm{~h}$, respectively. By agar screen, there were 23 (5.5\%) oxacillinresistant $S$. aureus strains after incubation for both 24 and 48 $\mathrm{h}$ and $162(65 \%)$ oxacillin-resistant coagulase-negative staphylococci at both time intervals. For $S$. aureus strains, there were no discrepancies between MicroScan and the agar screen at $24 \mathrm{~h}$; however, for five strains for which the oxacillin MIC was $2 \mu \mathrm{g} / \mathrm{ml}$ after $24 \mathrm{~h}$ of incubation the oxacillin MIC was $4 \mu \mathrm{g} / \mathrm{ml}$ after $48 \mathrm{~h}$ of incubation, whereas these strains remained susceptible by agar screen. The MIC for one $S$. aureus isolate increased from $1 \mu \mathrm{g} / \mathrm{ml}$ at $24 \mathrm{~h}$ to 16 $\mu \mathrm{g} / \mathrm{ml}$ at $48 \mathrm{~h}$, but the isolate remained susceptible by agar screen. For the coagulase-negative staphylococci, there was one discrepancy at $24 \mathrm{~h}$ between MicroScan and the agar screen; one strain for which oxacillin MICs were 1 and 8 $\mu \mathrm{g} / \mathrm{ml}$ at 24 and $48 \mathrm{~h}$, respectively, was resistant by oxacillin agar screen at $24 \mathrm{~h}$.

Thirteen isolates underwent additional testing by broth macrodilution (oxacillin with and without $4 \mu \mathrm{g}$ of clavulanic acid per ml) and disk diffusion (oxacillin and amoxicillinclavulanic acid). Moreover, the oxacillin agar screen was repeated with both Difco and BBL MHAs (Table 2). The macrodilution oxacillin MICs for all 10 strains of $S$. aureus were reduced at least fourfold by clavulanic acid, whereas only 2 of the 10 strains were susceptible to amoxicillinclavulanic acid by disk diffusion. Of the eight $S$. aureus strains for which the MicroScan oxacillin MIC was $2 \mu \mathrm{g} / \mathrm{ml}$ at $24 \mathrm{~h}$, five were intermediate and three were susceptible to oxacillin by disk diffusion. The remaining two strains of $S$. aureus, for each of which the oxacillin MIC was $1 \mu \mathrm{g} / \mathrm{ml}$ at $24 \mathrm{~h}$, increasing to 4 and $16 \mu \mathrm{g} / \mathrm{ml}$, respectively, at $48 \mathrm{~h}$, were susceptible to oxacillin by disk diffusion.

TABLE 1. Activity of oxacillin against staphylococci by MicroScan and oxacillin agar screen

\begin{tabular}{|c|c|c|c|c|c|c|}
\hline \multirow{3}{*}{ Organism and incubation time (h) ${ }^{a}$} & \multicolumn{6}{|c|}{ No. (\%) of strains } \\
\hline & \multicolumn{5}{|c|}{ With MicroScan MIC $(\mu \mathrm{g} / \mathrm{ml})$ : } & \multirow{2}{*}{$\begin{array}{l}\text { Resistant by oxacillin } \\
\text { agar screen }\end{array}$} \\
\hline & $<0.5$ & 1 & 2 & 4 & $>4$ & \\
\hline \multicolumn{7}{|l|}{ Staphylococcus aureus $(n=420)$} \\
\hline 24 & $368(87)$ & $21(5)$ & $8(2)$ & $1(0.2)$ & $22(5)$ & $23(5.5)$ \\
\hline 48 & $315(75)$ & $42(10)$ & $33(8)$ & $6(1.4)$ & $24(5.7)$ & $23(5.5)$ \\
\hline \multicolumn{7}{|c|}{ Coagulase-negative staphylococci $(n=248)$} \\
\hline 24 & $85(34)$ & $1(0.4)$ & $1(0.4)$ & $2(0.8)$ & $159(64)$ & $162(65)$ \\
\hline 48 & $85(34)$ & 0 & $1(0.4)$ & $1(0.4)$ & $161(65)$ & $162(65)$ \\
\hline
\end{tabular}

\footnotetext{
${ }^{a}$ Incubation was at $35^{\circ} \mathrm{C}$ for the first $24 \mathrm{~h}$ and $30^{\circ} \mathrm{C}$ for the second $24 \mathrm{~h}$.
} 
TABLE 2. Activity of oxacillin against 13 borderline or intermediately susceptible staphylococci as determined by MicroScan, broth macrodilution, disk diffusion, and agar screen ${ }^{\prime \prime}$

\begin{tabular}{|c|c|c|c|c|c|c|c|c|}
\hline \multirow{3}{*}{ Organism and isolate no. } & \multicolumn{4}{|c|}{$\operatorname{MIC}(\mu \mathrm{g} / \mathrm{ml})$} & \multirow{2}{*}{\multicolumn{2}{|c|}{ Disk diffusion ${ }^{b}$}} & \multirow{2}{*}{\multicolumn{2}{|c|}{ Oxacillin agar screen ${ }^{c}$}} \\
\hline & \multicolumn{2}{|c|}{ MicroScan } & \multicolumn{2}{|c|}{ Macrodilution } & & & & \\
\hline & $24 \mathrm{~h}$ & $48 \mathrm{~h}$ & NC & CLAV & OX & A-C & BBL & Difco \\
\hline \multicolumn{9}{|l|}{ Staphylococcus aureus } \\
\hline 142 & 2 & 2 & 3.12 & 0.78 & 1 & $\mathbf{S}$ & $\mathbf{S}$ & $\mathrm{S}$ \\
\hline 528 & 2 & 4 & 1.56 & 0.39 & I & $\mathbf{R}$ & $\mathbf{S}$ & $\mathrm{S}$ \\
\hline 511 & 2 & 4 & 1.56 & 0.39 & l & $\mathbf{R}$ & $\mathbf{S}$ & $\mathrm{S}$ \\
\hline 597 & 2 & 4 & 1.56 & 0.39 & 1 & $\mathbf{R}$ & $S$ & $S$ \\
\hline 601 & 2 & 2 & 1.56 & 0.39 & S & $S$ & $S$ & $\mathrm{~S}$ \\
\hline 628 & 2 & 4 & 1.56 & 0.39 & $\mathbf{S}$ & $\mathbf{R}$ & $\mathbf{S}$ & $\mathbf{S}$ \\
\hline 649 & 2 & 4 & 1.56 & 0.1 & $\mathbf{S}$ & $\mathbf{R}$ & $\mathbf{S}$ & $\mathbf{S}$ \\
\hline 193 & 2 & 2 & 1.56 & 0.39 & I & $\mathbf{R}$ & $S$ & $S$ \\
\hline 143 & 1 & 4 & 1.56 & 0.39 & $\mathbf{S}$ & $\mathbf{R}$ & $\mathbf{S}$ & $\mathrm{S}$ \\
\hline 409 & 1 & 16 & 1.56 & 0.39 & $\mathrm{~S}$ & $\mathbf{R}$ & $\mathrm{S}$ & $\mathbf{S}$ \\
\hline \multicolumn{9}{|c|}{ Coagulase-negative staphylococci } \\
\hline 66 & 4 & 16 & 3.12 & 0.78 & I & $\mathbf{S}$ & $\mathbf{R}$ & $\mathbf{R}$ \\
\hline 402 & 1 & 8 & 1.56 & 1.56 & I & $S$ & $\mathbf{R}$ & $\mathbf{R}$ \\
\hline 588 & 2 & 2 & 1.56 & 0.78 & 1 & S & S & 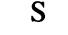 \\
\hline
\end{tabular}

a Abbreviations: NC, no clavulanic acid; CLAV, with clavulanic acid: OX, oxacillin, A-C, amoxicillin-clavulanic acid; I, intermediate; S, susceptible; R, resistant.

${ }^{b}$ Zone diameter interpretation: for oxacillin, $\geq 13 \mathrm{~mm}=$ susceptible; $\leq 10 \mathrm{~mm}=$ resistant: 11 to $12 \mathrm{~mm}=$ intermediate; for amoxicillin-clavulanic acid, $\geq 20$ $\mathrm{mm}=$ susceptible: $\leq 19 \mathrm{~mm}=$ resistant.

${ }^{c}$ Results at 24 and $48 \mathrm{~h}$ of incubation were unchanged.

Of the three coagulase-negative staphylococci, the macrodilution oxacillin MIC for only one strain was reduced at least fourfold by clavulanic acid, whereas all three strains were susceptible to amoxicillin-clavulanic acid by disk diffusion. By disk diffusion, all three strains were intermediately susceptible to oxacillin, and corresponding MicroScan MICs at $24 \mathrm{~h}$ were 1,2 , and $4 \mu \mathrm{g} / \mathrm{ml}$. The MIC for the latter strain was reduced fourfold by clavulanic acid.

All $S$. aureus strains were susceptible by the oxacillin agar screen at 24 and $48 \mathrm{~h}$. Of the three coagulase-negative staphylococci, one (for which the oxacillin MIC was $2 \mu \mathrm{g} / \mathrm{ml}$ at 24 and $48 \mathrm{~h}$ ) was susceptible by the agar screen at 24 and $48 \mathrm{~h}$. The remaining two coagulase-negative staphylococci were resistant by agar screen at $24 \mathrm{~h}$. There were no discrepancies between BBL and Difco MHAs.

\section{DISCUSSION}

In our study, we found that for detection of oxacillinresistant $S$. aureus and oxacillin-resistant coagulase-negative staphylococci the present MicroScan MIC panels (containing oxacillin in broth supplemented with $2 \% \mathrm{NaCl}$ ) used according to manufacturer directions performed comparably to the oxacillin agar screen, which is one of the methods generally accepted as a reference procedure (19). Similar findings have been reported by others (Brenner et al., Abstr. Annu. Meet. Am. Soc. Microbiol. 1987). On the other hand, Coudron et al., who tested a much smaller number of isolates, found that, after incubation for $24 \mathrm{~h}$, MicroScan detected only $75 \%$ of methicillin-resistant $S$. aureus (5). The reason for this discrepancy is unclear.

Investigators have demonstrated enhanced detection of heteroresistant staphylococci after incubation for $48 \mathrm{~h}(4,6)$ and by incubation at $30^{\circ} \mathrm{C}(10)$, although without $2 \% \mathrm{NaCl}$ supplementation, after $24 \mathrm{~h}$ of incubation at $35^{\circ} \mathrm{C}$; therefore, if the MicroScan oxacillin MIC was $\leq 4 \mu \mathrm{g} / \mathrm{ml}$ and/or if the strain was susceptible by oxacillin agar screen, we incubated the isolate at $30^{\circ} \mathrm{C}$ for an additional $24 \mathrm{~h}$. By doing this, we found that by MicroScan significantly more $S$. aureus strains were oxacillin resistant at $48 \mathrm{~h}$ than at $24 \mathrm{~h}(P=0.0078$, McNemar's chi square). This is in contrast to what Brenner et al. observed (Abstr. Annu. Meet. Am. Soc. Microbiol. 1987); however, they incubated panels at $35^{\circ} \mathrm{C}$ for the entire $48 \mathrm{~h}$, which could account for the disparate results. In contrast to MicroScan results, there was no change in the detection of oxacillin-resistant $S$. aureus or coagulase-negative staphylococci at 24 and $48 \mathrm{~h}$ by the oxacillin agar screen.

Prolonged incubation of staphylococci, however, can be problematic, because strains for which the oxacillin MIC is 1 to $2 \mu \mathrm{g} / \mathrm{ml}$ at $24 \mathrm{~h}$ may appear resistant to oxacillin at $48 \mathrm{~h}$ by virtue of the production of excessive amounts of $\beta$-lactamase, as described by McDougal and Thornsberry (14). Moreover, questions addressing the issue of appropriate laboratory and clinical interpretation of staphylococci for which the oxacillin MIC is 2 or $4 \mu \mathrm{g} / \mathrm{ml}$ have recently been raised. Therefore, in an attempt to accurately characterize our isolates that appeared to be borderline susceptible or intermediately susceptible to oxacillin, we examined strains that could be classified as such in greater depth. To detect $\beta$-lactamase production, we tested susceptibility to oxacillin with and without clavulanic acid by broth macrodilution, as well as susceptibility to amoxicillin-clavulanic acid by disk diffusion. We performed oxacillin disk diffusion testing. And because BBL MHA has been shown to detect more intrinsic oxacillin-resistant staphylococci than Difco MHA at $24 \mathrm{~h}$ (11), we repeated the oxacillin agar screen with both media.

The discrepancies we observed between the two methods used to detect $\beta$-lactamase production point out the inability of amoxicillin-clavulanic acid disk diffusion to reliably differentiate excessive $\beta$-lactamase-producing strains of staphylococci from truly intermediately susceptible strains. By disk diffusion, only 2 of $10 \mathrm{~S}$. aureus strains and all 3 coagulase-negative staphylococci would be considered to be $\beta$-lactamase producers, since they appeared susceptible to amoxicillin-clavulanic acid. In contrast, by broth macrodilu- 
tion, all $10 S$. aureus strains and only 1 of the 3 coagulasenegative staphylococci proved to be $\beta$-lactamase producers, since the oxacillin MIC for these strains was reduced at least fourfold with the addition of clavulanic acid. Although none of our $S$. aureus strains were truly intermediately susceptible, at least one and possibly two strains of coagulasenegative staphylococci could be classified as such. The one coagulase-negative staphylococcal strain (no. 402) that we believe could be called intermediately susceptible was our only isolate yielding a discrepancy between MicroScan and the oxacillin agar screen at $24 \mathrm{~h}$. This particular strain was susceptible to oxacillin by MicroScan at $24 \mathrm{~h}(\mathrm{MIC}, 1 \mu \mathrm{g} / \mathrm{ml})$ but resistant at $48 \mathrm{~h}(\mathrm{MIC}, 8 \mu \mathrm{g} / \mathrm{ml})$. It was intermediately susceptible to oxacillin by disk diffusion and resistant by the agar screen, and its macrodilution oxacillin MIC was unaffected by clavulanic acid. For the possible intermediately susceptible coagulase-negative staphylococcus, the oxacillin MIC was $2 \mu \mathrm{g} / \mathrm{ml}$ at 24 and $48 \mathrm{~h}$ by MicroScan and the MIC was $1.56 \mu \mathrm{g} / \mathrm{ml}$ by broth macrodilution, which was reduced only twofold by clavulanic acid. It was intermediately susceptible to oxacillin by disk diffusion and susceptible by the agar screen. Given that intrinsically resistant or heterogeneous staphylococci are often multiply resistant to other antimicrobial agents, we reviewed the MicroScan antibiograms of the 13 strains that underwent additional testing to determine whether the susceptibility patterns would support our above-described classification. Only two strains demonstrated resistance to several antimicrobial agents. The coagulase-negative staphylococcus for which the 24-h oxacillin MIC was $4 \mu \mathrm{g} / \mathrm{ml}$ was resistant to erythromycin, gentamicin, tetracycline, and trimethoprim-sulfamethoxazole. One $S$. aureus strain (no. 193, for which the oxacillin MIC was 2 $\mu \mathrm{g} / \mathrm{ml}$ at 24 and $48 \mathrm{~h}$ ) was resistant to clindamycin, erythromycin, and gentamicin. All other strains were either susceptible to all antibiotics except penicillin or resistant to one agent in addition to penicillin. On the basis of these antibiograms, we could not discern a difference between intermediately susceptible and $\beta$-lactamase-producing strains. However, the number of strains we examined was small, and it is possible that with larger numbers a distinction between these types of strains would be apparent.

Because appropriate therapy for borderline-susceptible and intermediately susceptible staphylococci is a significant issue, we attempted to review the charts of the nine patients from whom these 13 strains were recovered. Only six charts could be retrieved, and none of these patients received oxacillin, nafcillin, or methicillin. Only two isolates, one $S$. aureus strain and one coagulase-negative staphylococcus, both of which were recovered from blood, were considered clinically significant, and both patients were treated with vancomycin.

Oxacillin disk diffusion did not provide information helpful in distinguishing intermediately oxacillin-susceptible staphylococci from those capable of excessive $\beta$-lactamase production. Moreover, we found no difference between BBL and Difco MHAs, although the number of isolates included in the agar comparison was much too small to allow us to reach a significant conclusion.

In summary, we found that, compared with the oxacillin agar screen, MicroScan reliably detected oxacillin-resistant staphylococci after incubation for $24 \mathrm{~h}$. Moreover, in our opinion, incubation of MicroScan panels should not be extended beyond $24 \mathrm{~h}$. With the exception of one coagulasenegative staphylococcus, prolonged incubation resulted in an interpretation of oxacillin resistance, which in actuality was a consequence of excessive $\beta$-lactamase production.

\section{ACKNOWLEDGMENTS}

We thank Michelle Fisher and Karen Brodersen for their secretarial assistance.

\section{LITERATURE CITED}

1. Aldridge, K. E., A. Janney, C. V. Sanders, and R. L. Marier. 1983. Interlaboratory variation of antibiograms of methicillinresistant and methicillin-susceptible Staphylococcus aureus strains with conventional and commercial testing systems. J. Clin. Microbiol. 18:1226-1236.

2. Archer, G. L. 1978. Antimicrobial susceptibility and selection of resistance among Staphylococcus epidermidis isolates recovered from patients with infections of indwelling foreign devices. Antimicrob. Agents Chemother. 14:353-359.

3. Boyce, J. M., L. S. Lytle, and D. A. Walsh. 1984. Detection of methicillin-resistant Staphylococcus aureus by microdilution and disk elution susceptibility systems. J. Clin. Microbiol. 20: 1068-1075.

4. Bulger, R. J. 1967. A methicillin-resistant strain of Staphylococcus aureus. Clinical and laboratory experience. Ann. Intern. Med. 67:81-89.

5. Coudron, P. E., D. L. Jones, H. P. Dalton, and G. L. Archer. 1986. Evaluation of laboratory tests for detection of methicillinresistant Staphylococcus aureus and Staphylococcus epidermidis. J. Clin. Microbiol. 24:764-769.

6. Ericsson, J. W., and J. C. Sherris. 1971. Antibiotic sensitivity testing. Report of an international collaborative study. Acta Pathol. Microbiol. Scand. Suppl. 217:1-90.

7. Friedman, L. E., A. E. Brown, D. R. Miller, and D. Armstrong. 1984. Staphylococcus epidermidis septicemia in children with leukemia and lymphoma. Am. J. Dis. Child. 138:715-719.

8. Haley, R. W., A. W. Hightower, R. F. Khabbaz, C. Thornsberry, W. J. Martone, J. R. Allen, and J. M. Hughes. 1982. The emergence of methicillin-resistant Staphylococcus aureus infections in United States hospitals. Ann. Intern. Med. 97:297-308.

9. Hansen, S. L., and P. K. Freedy. 1984. Variation in the abilities of automated, commercial, and reference methods to detect methicillin-resistant (heteroresistant) Staphylococcus aureus. J. Clin. Microbiol. 20:494-499.

10. Hewitt, J. A., A. W. Coe, and M. T. Parker. 1969. The detection of methicillin resistance in Staphylococcus aureus. J. Med. Microbiol. 2:443-456.

11. Hindler, J. A., and C. B. Inderlied. 1985. Effect of the source of Mueller-Hinton agar and resistance frequency on the detection of methicillin-resistant Staphylococcus aureus. J. Clin. Microbiol. 21:205-210.

12. Karchmer, A. W., G. L. Archer, and W. E. Dismukes. 1983. Staphylococcus epidermidis causing prosthetic valve endocarditis: microbiologic and clinical observations as guides to therapy. Ann. Intern. Med. 98:447-455.

13. Klimek, J. J., F. J. Marsik, R. C. Bartlett, B. Wier, P. Shea, and R. Quintiliani. 1976. Clinical, epidemiologic and bacteriologic observations of an outbreak of methicillin-resistant Staphylococcus aureus at a large community hospital. Am. J. Med. 61: $340-345$.

14. McDougal, L. K., and C. Thornsberry. 1986. The role of $\beta$-lactamase in staphylococcal resistance to penicillinase-resistant penicillins and cephalosporins. J. Clin. Microbiol. 23:832839.

15. National Committee for Clinical Laboratory Standards. 1984. Performance standards for antimicrobic disc susceptibility tests, 3rd ed. Approved standard M2-A3. National Committee for Clinical Laboratory Standards, Villanova, $\mathrm{Pa}$.

16. National Committee for Clinical Laboratory Standards. 1985. Standard methods for dilution antimicrobial tests for bacteria which grow aerobically. Approved standard M7-A. National Committee for Clinical Laboratory Standards, Villanova, Pa.

17. Robinson, A., J. E. Mortensen, and S. B. Griffith. 1986. Susceptibility testing of methicillin-resistant Staphylococcus aureus with three commercial microdilution systems. Diagn. Microbiol. Infect. Dis. 5:245-253.

18. Thornsberry, C., J. Q. Caruthers, and C. N. Baker. 1973. Effect 
of temperature on the in vitro susceptibility of Staphylococcus aureus to penicillinase-resistant penicillins. Antimicrob. Agents Chemother. 4:263-269.

19. Thornsberry, C., and L. K. McDougal. 1983. Successful use of broth microdilution in susceptibility tests for methicillin-resistant (heteroresistant) staphylococci. J. Clin. Microbiol. 18: 1084-1091.

20. Wade, J. C., S. C. Schimpfi, K. A. Newman, and P. H. Wiernik. 1982. Staphylococcus epidermidis: an increasing cause of infection in patients with granulocytopenia. Ann. Intern. Med. 97: 503-508.
21. Winston, D. J., D. V. Dudnick, M. Chapin, W. G. Ho, R. P. Gale, and W. J. Martin. 1983. Coagulase-negative staphylococcal bacteremia in patients receiving immunosuppressive therapy. Arch. Intern. Med. 143:32-36.

22. Woods, G. L., G. S. Hall, I. Rutherford, K. J. Pratt, and C. C. Knapp. 1986. Detection of methicillin-resistant Staphylococcus epidermidis. J. Clin. Microbiol. 24:349-352.

23. Yu, P. K. W., and J. A. Washington II. 1985. Identification of aerobic and facultatively anaerobic bacteria, p. 135-136. In J. A. Washington II (ed.), Laboratory procedures in clinical microbiology, 2nd ed. Springer-Verlag, New York. 